\title{
19. Soziale Einbettung und freiwilliges Engagement
}

\author{
Oliver Huxhold \& Nicole Hameister
}

\section{Kernaussagen}

Sozial gut eingebettete Menschen sind anteilig häufiger engagiert. Eine gute Netzwerkqualität, das Vorhandensein sozialer Unterstützung außerhalb des eigenen Haushaltes und ein hohes soziales Vertrauen gehen unabhängig und signifikant mit einer erhöhten Wahrscheinlichkeit einher, sich zu engagieren.

Das Vorhandensein sozialer Unterstützung ist insbesondere für Frauen wichtig, um eine freiwillige Tätigkeit auszuüben. Die besondere Wichtigkeit von sozialer Unterstützung für das Engagement von Frauen ist vermutlich eine Folge der schlechteren Ressourcenausstattung von Frauen im Vergleich zu Männern.

Für das Engagement von Jüngeren sowie Älteren spielt das Vertrauen in andere Menschen eine größere Rolle als bei Personen mittleren Alters. Für Menschen, die jünger als 30 Jahre oder älter als 65 Jahre sind, spielt das Ausmaß des Vertrauens für das Engagement eine besondere Rolle.

Je höher die Gemeinschaftlichkeit im Wohnviertel ist, desto mehr Personen gehen einer freiwilligen Tätigkeit nach. Nicht nur die individuellen sozialen Ressourcen beeinflussen den Anteil engagierter Menschen, der soziale Zusammenhalt in der unmittelbaren Wohnumgebung zeigt Einflüsse auf das Engagement, die darüber hinausgehen.

Das Engagement kann die individuelle soziale Einbettung verbessern. Menschen, die lange engagiert sind, haben häufiger eine hohe subjektiv empfundene Netzwerkqualität als Personen, die noch nicht so lange freiwillig tätig sind.

\subsection{Einleitung}

Freiwilliges Engagement ist fest eingebunden in ein breites Spektrum sozialer Beziehungen und anderer sozialer Aktivitäten (Morrow-Howell, Hong \& Tang 2009). Die Gesamtheit der individuellen sozialen Kontakte, des persönlichen Netzwerkes und gemeinschaftlicher Unternehmungen wird in der Literatur häufig unter den Begriffen soziale Integration oder soziale Einbettung zusammengefasst (Huxhold, Fiori \& Windsor 2013). Soziale Einbettung ist dabei als ein Konstrukt aus unterschiedlichen Facetten zu verstehen (Antonucci, Ajrouch \& Birditt 2014), die sich unter anderem auch darauf auswirken können, ob Menschen ein Engagement aufnehmen beziehungsweise aufrechterhalten. Eine Facette stellt die Anzahl von Personen im individuellen sozialen Netzwerk dar. Also das Netz aus Familienmitgliedern, Freundinnen, Freunden, Nachbarinnen, Nachbarn und Bekannten, mit denen das Individuum in Beziehung steht. Oft stammt der Anreiz, ein Engagement aufzunehmen aus genau diesem unmittelbaren Netzwerk, denn Menschen erfahren hier oft von Gelegenheiten sich zu engagieren oder werden ganz direkt auf-

(C) Der/die Autor(en) 2017

J. Simonson et al. (Hrsg.), Freiwilliges Engagement in

Deutschland, Empirische Studien zum bürgerschaftlichen

Engagement, DOI 10.1007/978-3-658-12644-5_20 
gefordert, sich für ein konkretes Engagement zu entscheiden (Tang 2006; siehe Kapitel 15). Generell hat die Forschung immer wieder gezeigt, dass die Größe des sozialen Netzwerkes nicht allein für eine hohe soziale Eingebundenheit sorgt. Die Qualität der sozialen Beziehungen in diesem Netz ist genauso wichtig (Litwin 2011). Menschen, die sowohl über viele als auch über gute soziale Kontakte verfügen, sind daher vermutlich auch eher freiwillig engagiert als Menschen, die weniger gut eingebunden sind. Ein starkes soziales Netzwerk bietet aber auch soziale Unterstützung, denn hier finden Menschen Hilfe auch in schwierigen Zeiten. Dies kann sich in der Entlastung von bestimmten Aufgaben (wie beispielsweise der Kinderbetreuung oder der Pflege von Angehörigen) äußern, was Engagierten wiederum hilft, ihrer freiwilligen Tätigkeit nachzugehen. Zudem ist das soziale Netzwerk eng verknüpft mit dem generellen Vertrauen in andere Menschen - in der Forschung oft als ,social trust ${ }^{\star}$ oder ,soziales Vertrauen' bezeichnet. Dieses Vertrauen speist sich aus positiv erlebten Beziehungen zu Anderen und bildet damit die Grundlage für den Wunsch sich für die Gemeinschaft $\mathrm{zu}$ engagieren (Svendsen, Svendsen \& Graeff 2012; van Ingen \& Bekkers 2013). Die individuelle soziale Einbettung kann also auf vielfältige Weise Engagement beeinflussen, da ihre verschiedenen Facetten unterschiedliche Ressourcen bereitstellen, die das Individuum nutzen kann, um sich zu engagieren.

Doch individuelle soziale Ressourcen stellen nicht notwendigerweise den einzigen sozialen Einfluss auf die Entscheidungen dar, ein Engagement auszuüben. Menschliches Verhalten entsteht generell aus einem Wechselspiel zwischen individuellen Charakteristiken und Ressourcen und der Gelegenheitsstruktur, die die Umwelt bietet (Bronenbrenner 1977). Aus diesem Grund wollen wir in diesem Kapitel nicht nur die sozialen Ressourcen eines Menschen betrachten, sondern auch den räumlichen Lebenskontext einbeziehen, wenn wir die Zusammenhänge zwischen sozialer Einbettung und dem Engagement untersuchen. Deshalb werden wir analysieren, ob das soziale Gefüge im Wohnviertel ebenfalls die Entscheidung beeinflusst, eine freiwillige Tätigkeit auszuüben. Ein gutes soziales Miteinander könnte sowohl Möglichkeiten in der Wohnumgebung schaffen, um sich zu engagieren, als auch Hemmnissen entgegenwirken, die ein Engagement erschweren wie beispielsweise die Erreichbarkeit von Einrichtungen.

Um sich den Zusammenhängen von sozialer Einbettung angemessen widmen zu können, ist es wichtig, Unterschiede zwischen den Geschlechtern und Altersgruppen zu berücksichtigen. Studien zeigen, dass beispielsweise Frauen in der Regel eine bessere soziale Einbettung als Männer aufweisen (Ajrouch, Blandon \& Antonucci 2005). Auch das Alter spielt eine Rolle: Entgegen der öffentlichen Erwartungen ist das hohe Erwachsenenalter jedoch nicht zwingend durch eine verminderte soziale Einbettung geprägt. Eine Meta-Studie zeigt beispielsweise, dass die Größe nicht-familialer Netzwerke mit dem Alter kleiner werden (Wrzus, Hänel, Wagner \& Neyer 2013). Familiale soziale Kontakte nehmen hingegen deutlich weniger stark ab. Fragt man ältere Menschen nach der subjektiv erlebten Qualität ihrer sozialen Beziehungen, so schätzen sie diese im Allgemeinen positiver ein als Jüngere (Scheibe \& Carstensen 2010).

Über den Aspekt der Gruppenunterschiede in der sozialen Einbettung hinaus, gibt es noch einen weiteren Gesichtspunkt, der dafür spricht, dass sich der Zusammenhang zwischen unterschiedlichen Facetten der sozialen Einbettung und dem Engagement zwischen gesellschaftlichen Gruppen unterscheidet. Zum Beispiel könnten besonders für Frauen soziale Ressourcen und ein gutes soziales Verhältnis im Wohnviertel wichtige Voraussetzungen für Engagement sein, da sie häufiger als Männer einer doppelten Belastung durch Beruf und Familie ausgesetzt sind. Diese besondere Situation kann dazu führen, dass insbesondere soziale Unterstützung für das Engagement von Frauen entscheidender ist als für die freiwilligen Tätigkeiten von Männern. Der möglicherweise größere Bedarf an Unterstützung lässt sie in besonderem 
Maße auf das persönliche Netzwerk angewiesen sein. Zudem könnte die Bedeutung sozialer Einbettung für das Engagement auch mit dem Alter zunehmen. Gerade der Einfluss der unmittelbaren Wohnumgebung könnte für ältere Menschen wichtig sein, denn diese sind möglicherweise gesundheitsbedingt in ihrer Mobilität eingeschränkt (Dury, Willems, De Witte, De Donder, Buffel \& Verté 2014).

Der Freiwilligensurvey bildet die individuelle soziale Einbettung insbesondere in drei wichtigen Facetten ab: Subjektive Netzwerkqualität, soziale Unterstützung und soziales Vertrauen. Zunächst werten wir den Zusammenhang des Engagements mit den drei erfassten Aspekten individueller sozialer Einbettung deskriptiv aus. Unsere Leithypothese lautet dabei, dass Menschen, die sich besser sozial integriert fühlen, auf die Hilfe anderer zurückgreifen können und ein höheres soziales Vertrauen aufweisen, eher engagiert sind als Personen, die nicht gut eingebettet sind. Bei der Untersuchung dieser Zusammenhänge fokussieren wir, wie oben ausgeführt, auf Gruppenunterschiede und beantworten die Frage, ob soziale Ressourcen wichtiger für das Engagement von Frauen und älteren Personen sind als für Männer und jüngere Menschen. Ein weiterer Aspekt, den wir in diesem Kapitel beleuchten, behandelt den Einfluss des unmittelbaren Wohnumfelds für die Aufnahme einer freiwilligen Tätigkeit. Auf der empirischen Ebene ist die Wahrnehmung der eigenen sozialen Einbettung vermutlich mit der Bewertung des sozialen $\mathrm{Zu}$ sammenhalts in der Wohnumgebung korreliert. Mit anderen Worten: Menschen, die ihre eigene soziale Einbettung als gut einschätzen, werden wahrscheinlich auch den Zusammenhalt im Wohnviertel positiv beurteilen. Zusätzlich ist es durchaus möglich, dass Personen im individuellen Netzwerk aus der Nachbarschaft stammen. Aus diesen Gründen untersuchen wir in einer multivariaten Analyse, in wie weit das soziale Gefüge im Wohnviertel das Engagement vorhersagt, wenn sowohl die Einflüsse der individuellen sozialen Einbettung als auch des Geschlechts, des Alters und des Bildungsniveaus kontrolliert werden.

Eine gute soziale Einbettung kann also ein freiwilliges Engagement begünstigen, aber auch der umgekehrte Zusammenhang ist in diesem Kontext denkbar und plausibel. In der Literatur zum Thema soziale Einbettung und Engagement wird zum Beispiel häufig argumentiert, dass es gerade die mit dem freiwilligen Engagement verbundenen Möglichkeiten zum sozialen Austausch sind, die den besonderen Reiz dieser Tätigkeiten ausmachen. Empirische Arbeiten, die untersuchen, ob freiwillig engagierte Menschen tatsächlich besser sozial integriert sind, weil ihnen freiwillige Tätigkeiten Möglichkeiten zum Aufbau sozialer Kontakte bieten, sind bislang relativ rar. Es gibt jedoch ein paar Hinweise, die dafür sprechen, dass Engagierte gerade das Gemeinschaftsgefühl, das ihnen ihre freiwillige Betätigung bietet, sehr zu schätzen wissen (Goth \& Småland 2014; Son \& Wilson 2012). Diese Befunde sind auch aus einem anderen Blickwinkel interessant: Ein Gefühl der Gemeinschaftlichkeit, das mit der Überzeugung einhergeht, dass Menschen im Allgemeinen vertrauenswürdig und fair sind, kann dazu führen, dass sich die Qualität des individuellen sozialen Netzwerkes auch außerhalb des Engagements erhöht (Wilson \& Musick 1997). In unserer empirischen Analyse lässt sich nur schwer trennen, ob freiwilliges Engagement tatsächlich $\mathrm{zu}$ einer verbesserten sozialen Einbettung führt oder ob vielmehr Menschen, die gut sozial eingebunden sind, sich eher engagieren. Aus diesem Grund werden wir in weiteren multivariaten Analysen untersuchen, ob auch die Dauer des bisherigen Engagements etwas über die individuelle soziale Einbettung aussagt. Denn wenn das Engagement hilft, soziale Beziehungen aufzubauen und zu pflegen, dann sollte sich die individuelle soziale Einbettung stetig verbessern je länger man es ausübt.

Im Freiwilligensurvey 2014 untersuchen wir den Zusammenhang zwischen sozialer Einbettung und dem Engagement mit fünf Fragestellungen: 
1. Unterscheiden sich Menschen mit unterschiedlich hoher Netzwerkqualität, mit oder ohne Unterstützungspotenzial außerhalb des Haushaltes, allgemeinem Vertrauen und unterschiedlicher Güte in der sozialen Wohnumgebung in ihrem Engagement?

2. Weisen Netzwerkqualität, Unterstützungspotenzial, allgemeines Vertrauen und unterschiedliche Güte in der sozialen Wohnumgebung in verschiedenen gesellschaftlichen Gruppen (nach Geschlecht und Alter) unterschiedliche Zusammenhänge mit Engagement auf?

3. Ist die Bedeutung von Netzwerkqualität, Unterstützungspotenzial und allgemeinem

\subsection{Datengrundlage}

Die in diesem Kapitel berichteten deskriptiven Befunde basieren auf gewichteten Analysen. Für die Gewichtung wurden in allen Erhebungsjahren dieselben Gewichtungsmerkmale berücksichtigt. Dabei handelt es sich um die Designgewichtung, die sich auf die Auswahlwahrscheinlichkeit einer Person bezieht (Haushaltsgröße, Zahl der Festnetznummern und, nur für das Jahr 2014, auch die Zahl der Mobilfunknummern) und Gewichtungsmerkmale, die Abweichungen der Stichprobenverteilung von der Grundgesamtheit in bestimmten Dimensionen ausgleichen (Bundesland, Gemeindegrößenklassen, Geschlecht, Altersgruppen). Von einer Hinzunahme weiterer Gewichtungsmerkmale wie etwa des Bildungsstandes haben wir abgesehen, um die Vergleichbarkeit mit den bisher veröffentlichten Berichten zu erhalten.

Subjektive Netzwerkqualität: Im Freiwilligensurvey 2014 werden alle Befragten um die subjektive Einschätzung ihrer Netzwerkqualität gebeten, mit folgendem Wortlaut:
Vertrauen für freiwilliges Engagement auch dann nachweisbar, wenn andere Aspekte der Lebenssituation von Menschen berücksichtigt werden (multivariate Betrachtung)?

4. Steht die unterschiedliche Qualität in der sozialen Wohnumgebung mit dem Engagement in Zusammenhang, wenn Unterschiede in der individuellen sozialen Einbettung und andere Aspekte der Lebenssituation von Menschen berücksichtigt werden (multivariate Betrachtung)?

5. Steht die Dauer der freiwilligen Tätigkeit mit der Netzwerkqualität, dem Unterstützungspotenzial und dem allgemeinen Vertrauen in Zusammenhang?

Inwieweit trifft die folgende Aussage auf Sie zu? Es gibt genügend Menschen, mit denen ich mich eng verbunden fühle.

Antwortkategorien: trifft voll und ganz zu; trifft eher zu; teils/teils; trifft eher nicht zu; trifft ganz und gar nicht $z u$

Für dieses Kapitel fassen wir die Ausprägungen ,trifft voll und ganz $\mathrm{zu}^{\prime}$ und ,trifft eher $\mathrm{zu}^{\prime} \mathrm{zu}$ ,hohe subjektive Netzwerkqualität' zusammen. 77,2 Prozent der Befragten im Freiwilligensurvey fallen in diese Kategorie. Erwartungsgemäß ist dieser Anteil etwas niedriger bei Männern (74,1 Prozent) als bei Frauen (80,1 Prozent). Die Prozentsätze derjenigen Personen, die über eine hohe Netzwerkqualität verfügen, unterscheiden sich jedoch nicht signifikant zwischen den $\mathrm{Al}$ tersgruppen.

Potenzial für soziale Unterstützung: $\mathrm{Ob}$ die Befragten über ein hohes Potenzial für soziale Unterstützung verfügen, erfasst der Freiwilligensurvey 2014 mit folgender Frage: 
Wenn Sie mal Hilfe brauchen, zum Beispiel bei Besorgungen, kleineren Arbeiten oder der Betreuung von Kindern oder Kranken: Gibt es da Personen außerhalb Ihres Haushaltes, an die Sie sich unentgeltlich wenden können?

Die Antwortmöglichkeiten sind ,ja und ,neinc Im Folgenden werden alle positiven Antworten als ,Unterstützungspotenzial vorhanden' gewertet. 87,7 Prozent der Befragten verfügen über diese Form der Unterstützung. Bei Frauen liegt dieser Anteil um 1,1 Prozentpunkte höher als bei Männern. Weiterhin nimmt der Prozentsatz an Personen mit Unterstützungspotenzial außerhalb des Haushaltes leicht über die Altersgruppen hinweg ab. In der ältesten Gruppe verfügen etwas weniger Menschen (6,8 Prozentpunkte) über hohes Unterstützungspotenzial als in der jüngsten Altersgruppe.

Tabelle 19-1: Vorhandensein hoher subjektiver Netzwerkqualität 2014

\begin{tabular}{lc}
\hline & Hohe Netzwerkqualität \\
\hline Gesamt & 77,2 \\
\hline Frauen & 80,1 \\
\hline Männer & 74,1 \\
\hline 14 bis 29 Jahre & 76,5 \\
\hline 30 bis 49 Jahre & 77,0 \\
\hline 50 bis 64 Jahre & 78,3 \\
\hline 65 Jahre und älter & 76,9 \\
\hline
\end{tabular}

Quelle: FWS 2014, gewichtet, eigene Berechnungen (DZA). Basis: Alle Befragten ( $n=28.420$ ).

Dargestellt sind Anteile in Prozent.

Tabelle 19-2: Vorhandensein von Unterstützungspotenzial außerhalb des Haushaltes 2014

\begin{tabular}{lc}
\hline & Unterstützungspotenzial vorhanden \\
\hline Gesamt & 87,7 \\
\hline Frauen & 88,2 \\
\hline Männer & 87,1 \\
\hline 14 bis 29 Jahre & 90,9 \\
\hline 30 bis 49 Jahre & 88,6 \\
\hline 50 bis 64 Jahre & 87,2 \\
\hline 65 Jahre und älter & 84,1 \\
\hline
\end{tabular}

Quelle: FWS 2014, gewichtet, eigene Berechnungen (DZA). Basis: Alle Befragten $(n=28.472)$.

Dargestellt sind Anteile in Prozent. 
Soziales Vertrauen: Der Freiwilligensurvey 2014 verwendet eine in sozialwissenschaftlichen Umfragen übliche Frageformulierung zur Erhebung des sozialen Vertrauens:

Wie sehr stimmen Sie der folgenden Aussage zu: Man kann den meisten Menschen vertrauen.

Die fünf abgestuften Antwortkategorien reichen von ,stimme voll und ganz zu' bis ,Stimme ganz und gar nicht zu'. Auch bei dieser Variablen fas- sen wir für die Analysen die Kategorien zusammen: stimme voll und ganz zu' sowie, stimme

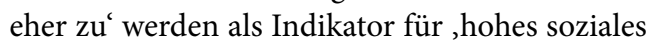
Vertrauen' interpretiert. Nur 32,4 Prozent der Befragten fallen in diese Kategorie. Dieser Anteil ist etwas höher bei Männern (33,6 Prozent) als bei Frauen (31,3 Prozent). In den beiden älteren Altersgruppen haben mehr Menschen ein hohes soziales Vertrauen als in den beiden jüngeren Gruppen.

Tabelle 19-3: Vorhandensein hohen sozialen Vertrauens 2014

\begin{tabular}{lc}
\hline & Hohes Vertrauen \\
\hline Gesamt & 32,4 \\
\hline Frauen & 31,3 \\
\hline Männer & 33,6 \\
\hline 14 bis 29 Jahre & 31,4 \\
\hline 30 bis 49 Jahre & 30,7 \\
\hline 50 bis 64 Jahre & 33,9 \\
\hline 65 Jahre und älter & 33,9 \\
\hline
\end{tabular}

Quelle: FWS 2014, gewichtet, eigene Berechnungen (DZA). Basis: Alle Befragten ( $n=28.472$ ).

Dargestellt sind Anteile in Prozent.

Sozialer Zusammenhalt im Wohnviertel: Alle Teilnehmerinnen und Teilnehmer des Freiwilligensurveys 2014 wurden gefragt:

Was würden Sie sagen: Wie gut ist der allgemeine Zusammenhalt in Ihrem Wohnviertel?

Die fünfstufige Skala umfasste die Ausprägungen ,sehr gut', ,eher gut', ,mittel', ,eher schlecht‘ sowie ,sehr schlecht'. Für dieses Kapitel fassen wir die Ausprägungen, sehr gut' und ,eher gut' zu ,hoher Zusammenhalt im Wohnviertel' zusammen. 61,1 Prozent der Befragten im Freiwilligensurvey fallen in diese Kategorie. Die Prozentsätze derjenigen Personen, die über einen hohen Zusammenhalt verfügen, unterscheiden sich nicht zwischen den Geschlechtern und Altersgruppen. 
Tabelle 19-4: Vorhandensein eines hohen Zusammenhalts im Wohnviertel 2014

\begin{tabular}{lc}
\hline & Hoher Zusammenhalt im Wohnviertel \\
\hline Gesamt & 61,1 \\
\hline Frauen & 61,2 \\
\hline Männer & 61,2 \\
\hline 14 bis 29 Jahre & 60,9 \\
\hline 30 bis 49 Jahre & 61,1 \\
\hline 50 bis 64 Jahre & 60,9 \\
\hline 65 Jahre und älter & 61,7 \\
\hline
\end{tabular}

Quelle: FWS 2014, gewichtet, eigene Berechnungen (DZA). Basis: Alle Befragten ( $n=27.704)$.

Dargestellt sind Anteile in Prozent.

Dauer des Engagements: Die Dauer der freiwilligen Tätigkeit wird bei allen Engagierten mit der Abfrage nach der Jahresanzahl erfasst:

Wie viele Jahre üben Sie diese Tätigkeit schon aus?

Für die Analysen in diesem Kapitel nehmen wir noch Modifizierungen an der Engagementdauer vor: All jene Engagierte, die angeben, dass sie ihre Tätigkeit erst seit weniger als einem Jahr beziehungsweise seit dem Jahr 2014 ausüben, erhalten den Wert 0 . Außerdem werden alle Jahreswerte über zehn zu zehn recodiert (dies betrifft etwa 14 Prozent der Befragten), unter anderem um eine vereinfachte Darstellung zu ermöglichen. Im Durchschnitt sind die Engagierten seit 6,3 Jahren engagiert.

Tabelle 19-5: Biografische Dauer des Engagements in Jahren (nur Engagierte) 2014

\begin{tabular}{lc}
\hline & Biografische Dauer in Jahren \\
\hline Gesamt & 6,3 \\
\hline Frauen & 6,0 \\
\hline Männer & 6,7 \\
\hline 14 bis 29 Jahre & 4,1 \\
\hline 30 bis 49 Jahre & 5,8 \\
\hline 50 bis 64 Jahre & 7,4 \\
\hline 65 Jahre und älter & 8,3 \\
\hline
\end{tabular}

Quelle: FWS 2014, gewichtet, eigene Berechnungen (DZA). Basis: Alle Engagierten ( $n=11.869$ ).

Dargestellt ist die durchschnittliche biografische Dauer in Jahren. 


\subsection{Stehen individuelle Aspekte der sozialen Einbettung mit dem Engagement im Zusammenhang?}

Subjektive Netzwerkqualität: Alle Teilnehmerinnen und Teilnehmer des Freiwilligensurveys werden gefragt, ob es genügend Menschen in ihrem Leben gibt, mit denen sie sich eng verbunden fühlen. Diese Frage erfasst die subjektiv empfundene Netzwerkqualität und bezieht sich gleichzeitig auf quantitative und qualitative Elemente der individuellen sozialen Einbettung. Beide Aspekte können wichtige Faktoren sein, die mitentscheiden, ob Menschen aus ihrem sozialen Netzwerk heraus angesprochen werden, ein Engagement aufzunehmen. In Abbildung 19-1 sind die Engagementraten für Personen zu sehen, die entweder eine niedrige oder eine hohe subjektive Netzwerkqualität haben, differenziert nach Geschlecht und Alter.

Der Vergleich der Engagementraten deutet einen starken Zusammenhang zwischen der Netzwerkqualität und dem Engagement an. Der Anteil der Engagierten ist bei Personen mit hoher subjektiver Netzwerkqualität um 18,2 Prozentpunkte höher als bei Menschen mit niedriger Netzwerkqualität. Dieser Unterschied ist ausgeprägter bei Männern, bei denen er 20,5 Prozentpunkte beträgt, als bei Frauen (16,7 Prozentpunkte). Die Stärke des Zusammenhanges unterscheidet sich nicht signifikant zwischen den Altersgruppen.

\section{Abbildung 19-1: Anteile Engagierter differenziert nach hoher und niedriger Netzwerkqualität 2014, gesamt, nach Geschlecht und nach Alter}

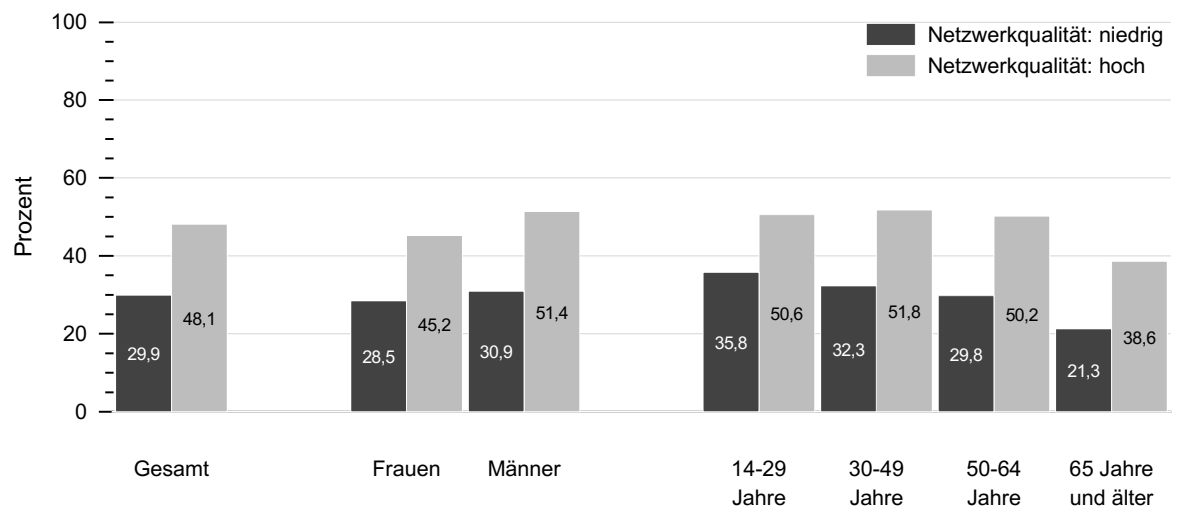

Quelle: FWS 2014, gewichtet, eigene Berechnungen (DZA). Basis: Alle Befragten ( $n=28.420$ ).

Potenzial für soziale Unterstützung: Das Potenzial für soziale Unterstützung hat generell einen Einfluss auf das Wohlbefinden im Lebensverlauf. Es gewinnt noch an Bedeutung, wenn es Alltagskrisen und Mehrfachbelastungen zu meistern gilt, in denen es eine wichtige Bewältigungsres- source darstellt. Für den Zusammenhang mit dem Engagement kann dies bedeuten, dass eine freiwillige Tätigkeit auch dann in persönlichen Krisenzeiten weiter ausgeübt werden kann, wenn die oder der Engagierte an anderer Stelle entlastet werden kann. 
In der Abbildung 19-2 wird der Prozentsatz von engagierten Personen dargestellt, wobei unterschieden wird zwischen Menschen, die von sich sagen, sie hätten Menschen außerhalb des eigenen Haushaltes, die sie unterstützen, und denen, die nicht darüber verfügen.

\section{Abbildung 19-2: Anteile Engagierter differenziert nach Unterstützungspotenzial 2014, gesamt, nach Geschlecht und nach Alter}

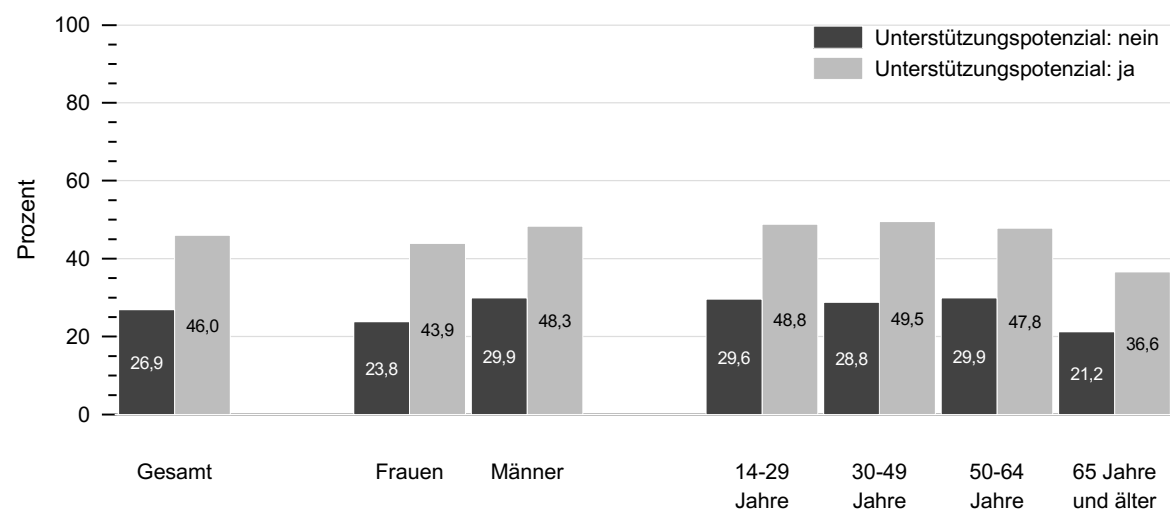

Quelle: FWS 2014, gewichtet, eigene Berechnungen (DZA). Basis: Alle Befragten ( $n=28.472$ ).

Erwartungsgemäß sind Menschen, die über Unterstützung außerhalb des Haushaltes verfügen, häufiger engagiert (46,0 Prozent) als die Menschen, die nicht darauf zurückgreifen können (26,9 Prozent). Dieser Unterschied ist bei Frauen (20,1 Prozentpunkte) ein wenig ausgeprägter als bei Männern (18,4 Prozentpunkte). Bei den beiden jüngeren Altersgruppen (20,3 Prozentpunkte Unterschied im Durchschnitt) fällt der Unterschied stärker aus als bei den beiden älteren Gruppen (16,7 Prozentpunkte Unterschied im Durchschnitt).

Soziales Vertrauen: Ein hohes Vertrauen in die Mitmenschen wird in verschiedenen Forschungstraditionen als positiv angesehen. Beispielsweise wird generalisiertes oder soziales Vertrauen sowohl in der wissenschaftlichen Diskussion als auch in der öffentlichen Debatte häufig als ein Marker für die Legitimität gesell- schaftlicher Institutionen betrachtet (Kaina 2004; Svendsen et al. 2012). In der Engagementdebatte wird Vertrauen deshalb häufig als Ursache oder Anreiz für freiwillige Tätigkeiten gesehen (van Ingen \& Bekkers 2013). Die Abbildung 19-3 zeigt Engagementraten unterschieden nach Personen, die von sich sagen, die meisten Menschen seien vertrauenswürdig, und denen, die dieses Vertrauen nicht haben.

Der Unterschied in den Engagementraten zwischen Menschen mit hohem beziehungsweise niedrigem sozialen Vertrauen beträgt 14,4 Prozentpunkte und unterscheidet sich nicht zwischen den Geschlechtern. In den beiden älteren Altersgruppen ist die Differenz stärker ausgeprägt (18,2 Prozentpunkte im Durchschnitt) als in den beiden jüngeren Gruppen (11,2 Prozentpunkte im Durchschnitt). 
Abbildung 19-3: Anteile Engagierter differenziert nach niedrigem und hohem sozialen Vertrauen 2014, gesamt, nach Geschlecht und nach Alter

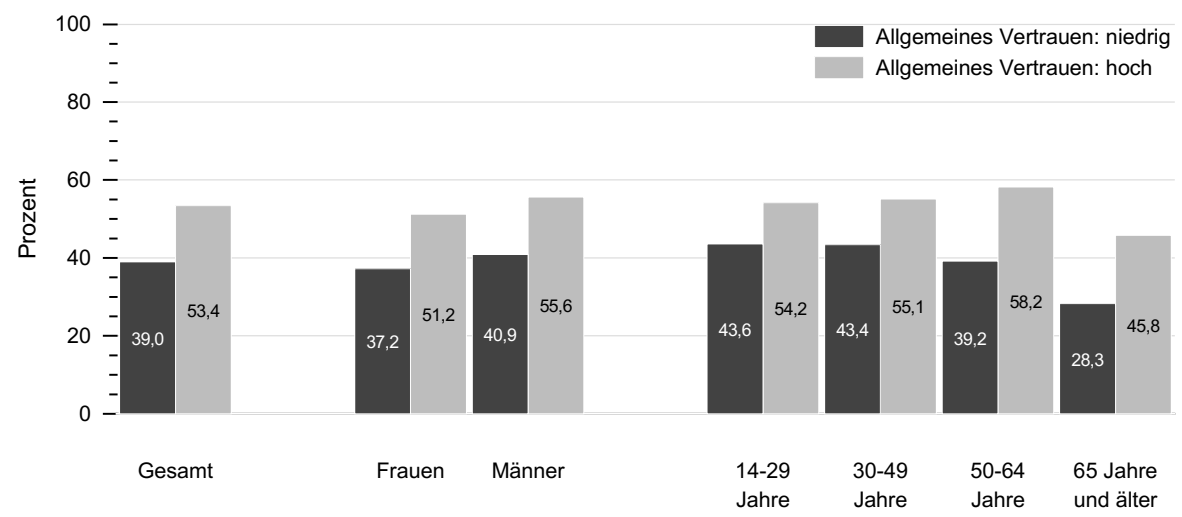

Quelle: FWS 2014, gewichtet, eigene Berechnungen (DZA). Basis: Alle Befragten $(n=28.568)$. 


\subsection{Welcher Zusammenhang besteht zwischen der sozialen Wohnumgebung und dem Engagement?}

Erhöht ein guter sozialer Zusammenhalt in der unmittelbaren Wohnumgebung die Wahrscheinlichkeit, eine freiwillige Tätigkeit aufzunehmen? Wir vermuteten, dass der Effekt des Wohnumfelds besonders für ältere Menschen ausgeprägt ist, weil diese eventuell aufgrund eingeschränkter Mobilität stärker auf nahegelegene Möglichkeiten zum Engagement angewiesen sein könnten.

Der Zusammenhalt im Wohnviertel steht mit der Wahrscheinlichkeit sich zu engagieren in Zusammenhang: 47,6 Prozent der Menschen, die einen hohen sozialen Zusammenhalt berichten, sind freiwillig tätig. Bei Personen, die das soziale Klima in ihrer unmittelbaren Wohnumgebung schlechter einstufen, engagieren sich nur 38,8 Prozent. Der Zusammenhang ist bei Männern und Frauen gleich stark. Entgegen unseren Erwartungen ist der Zusammenhang zwischen dem Zusammenhalt im Wohnviertel und dem Engagement in der ältesten Gruppe nicht stärker als bei Personen im Alter von 50 bis 64 Jahren. In der Gruppe der 14- bis 29-Jährigen ist der Zusammenhang zwischen dem sozialen Klima im Wohnviertel und der Anzahl der Personen, die eine freiwillige Tätigkeit ausüben allerdings geringer (4,5 Prozentpunkte Unterschied) als in den drei älteren Altersgruppen (9,1 Prozentpunkte Unterschied im Durchschnitt).

\section{Abbildung 19-4: Anteile Engagierter differenziert nach hohem und niedrigem Zusammenhalt im Wohnviertel 2014, gesamt, nach Geschlecht und nach Alter}

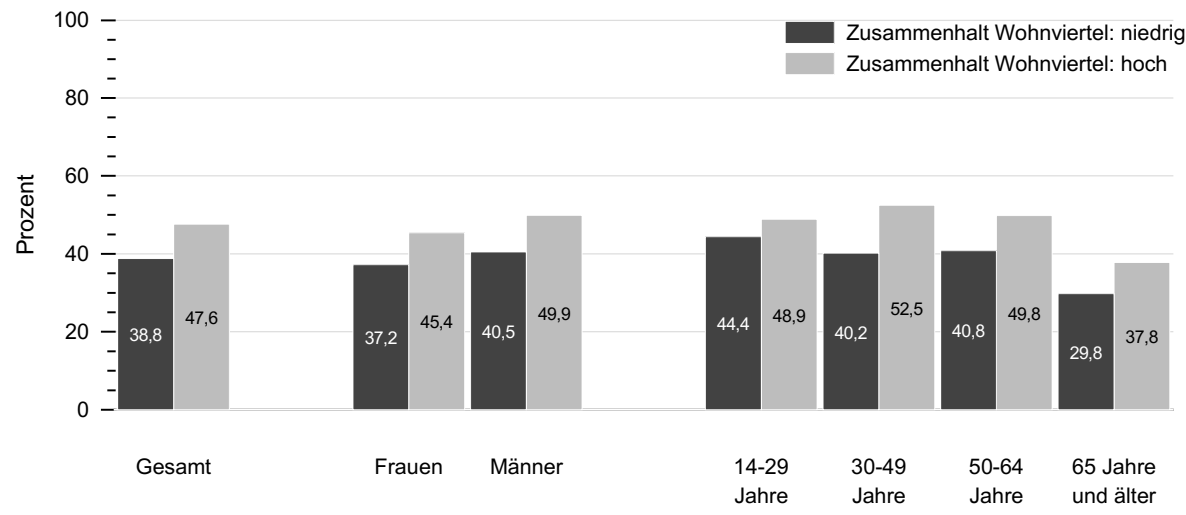

Quelle: FWS 2014, gewichtet, eigene Berechnungen (DZA). Basis: Alle Befragten $(n=27.704)$. 


\subsection{Steht die soziale Einbettung auch dann mit dem Engagement in Zusammenhang, wenn man Unterschiede in Ressourcen und Werthaltungen betrachtet?}

In den bisherigen Analysen in diesem Kapitel wurden die Zusammenhänge zwischen den drei Aspekten der sozialen Einbettung und dem Engagement dargestellt. In diesem Abschnitt gehen wir der Frage nach, ob die berichteten Zusammenhänge auch dann noch bestehen bleiben, wenn man für eine Reihe weiterer Einflussgrößen kontrolliert. So könnten sowohl die soziale Einbettung als auch das Engagement abhängig sein vom Bildungsstatus, finanziellen und gesundheitlichen Ressourcen oder einer solidarischen Werthaltung. Aus diesen Gründen überprüfen wir mit Hilfe einer multivariaten logistischen Regression, ob die zuvor berichteten Zusammenhänge sich auf diese genannten Drittvariablen zurückführen lassen. Die abhängige Variable in allen Modellen ist Engagement (ja/nein). Für jede der drei individuellen Aspekte sozialer Einbettung (Netzwerkqualität, Unterstützungspotenzial und soziales Vertrauen) wird jeweils ein Modell gerechnet; der jeweilige Aspekt stellt die Fokusvariable für das entsprechende Modell dar. In dieses Modell gehen als unabhängige Variablen jeweils eine der drei Fokusvariablen ein sowie Geschlecht und Alter der Personen und außerdem die Interaktionen zwischen Geschlecht und Alter mit den Fokusvariablen. Als Referenzgruppe für die Testung der Effekte wurde die Gruppe der Männer im Alter von 50 bis 64 Jahren mit mittlerem Bildungsniveau gewählt. Es wurde dabei diejenige Gruppe als Bezugspunkt gewählt, die im Alter und in der Bildung in einer mittleren Position liegt. Zudem wurde die Gruppe der Männer als Referenzgröße gewählt, um die Ergebnisse für die Gruppe der Frauen explizit darstellen zu können (wird die Referenzgruppe Frauen gewählt, kann kein Koeffizient für Frauen ausgewiesen werden). Als Kontrollvariablen gingen in die Analysen die folgenden Informa- tionen ein: Bildung, subjektives Einkommen, subjektive Gesundheit, Werthaltung Solidarität sowie Bundesland und Kontakt über Festnetz/ Mobilfunk. Berichtet werden unstandardisierte Koeffizienten, wobei ein negativer Koeffizient bedeutet, dass die Wahrscheinlichkeit sich zu engagieren, kleiner ist als in der Vergleichsgruppe, und ein positiver Koeffizient dafür steht, dass die Wahrscheinlichkeit größer ist. Berichtet werden nur signifikante Koeffizienten, nicht signifikante Koeffizienten werden mit drei Strichen dargestellt. In Tabelle 19-6 sind die Ergebnisse der logistischen Regressionen dargestellt.

Die multivariate Analyse der Netzwerkqualität bestätigt die deskriptiven Betrachtungen. Menschen mit guter Netzwerkqualität engagieren sich häufiger (Koeffizient 0,32), auch wenn man Unterschiede in gesundheitlichen und finanziellen Ressourcen und individuellen Werthaltungen berücksichtigt. Dieser Effekt ist über alle Altersgruppen gleich. Für Frauen ist die Netzwerkqualität weniger bedeutsam für die Wahrscheinlichkeit ein Engagement auszuüben als für Männer (Koeffizient -0,07).

Das Vorhandensein von Unterstützungspotenzial steht ebenfalls über den Effekt von Unterschieden in Ressourcen und Werthaltungen hinaus mit dem Engagement im Zusammenhang (Koeffizient 0,59). Dass der Zusammenhang zwischen dem Unterstützungspotenzial und Engagement bei Frauen stärker ist als bei Männern, so wie es sich in den deskriptiven Analysen andeutet, lässt sich multivariat nicht bestätigen. Vermutlich ist dieser Geschlechtereffekt also auf die niedrigere Ressourcenausstattung von Frauen zurückzuführen. Diese führt möglicherweise dazu, dass Frauen häufiger auf soziale Unterstützung zurückgreifen müssen, um ein Engagement $\mathrm{zu}$ beginnen oder es in schwierigen Zeiten bei- 
behalten zu können. Altersunterschiede im Ausmaß des Zusammenhanges zwischen Unterstützungspotenzial und Engagement gibt es nicht.

Menschen mit hohem sozialem Vertrauen engagieren sich häufiger als Personen mit geringerem Vertrauen (Koeffizient 0,24), selbst wenn wir Unterschiede bei den Ressourcen und Werthaltungen berücksichtigen. Vertrauen in andere Menschen hat für beide Geschlechter die gleiche
Wichtigkeit für das Engagementverhalten. Allerdings ist der Zusammenhang stärker bei Menschen ab 50 Jahren. Der Effekt ist weniger stark bei den 14- bis 29-Jährigen (Koeffizient -0,15) und den 30- bis 49-Jährigen (Koeffizient -0,10) als in der Vergleichsgruppe der 50- bis 64-Jährigen. Die älteste Altersgruppe unterscheidet sich dagegen nicht in der Stärke des Zusammenhanges von der zweitältesten Gruppe.

Tabelle 19-6: Ergebnisse logistischer Regressionen, Vorhersage freiwilligen Engagements durch drei Fokusvariablen (Netzwerkqualität, Unterstützungspotenzial, Soziales Vertrauen), Geschlecht, Alter, Bildung sowie Interaktionen der Fokusvariablen mit Geschlecht und Alter, 2014

\begin{tabular}{|c|c|c|c|}
\hline & $\begin{array}{l}\text { Netzwerk- } \\
\text { qualität }\end{array}$ & $\begin{array}{l}\text { Unterstützungs- } \\
\text { potenzial }\end{array}$ & $\begin{array}{l}\text { Soziales } \\
\text { Vertrauen }\end{array}$ \\
\hline Fokusvariable & $0,32 * * *$ & $0,59 * * *$ & $0,24 * * *$ \\
\hline \multicolumn{4}{|l|}{ Geschlecht (Referenz: Männer) } \\
\hline Frauen & $-0,24 * * *$ & $-0,32 * * *$ & $-0,20 * * *$ \\
\hline \multicolumn{4}{|l|}{ Alter (Referenz: 50 bis 64 Jahre) } \\
\hline 14 bis 29 Jahre & $-0,13^{* * *}$ & $-0,34^{*}$ & $-0,13^{* *}$ \\
\hline 30 bis 49 Jahre & $0,07^{*}$ & --- & $0,07^{*}$ \\
\hline 65 Jahre und älter & $-0,25^{* * *}$ & $-0,25^{*}$ & $-0,27 * * *$ \\
\hline \multicolumn{4}{|l|}{ Bildung (Referenz: Mittlere Bildung) } \\
\hline Noch Schüler/in & $0,51 * * *$ & $0,56 * * *$ & $0,51 * * *$ \\
\hline Niedrige Bildung & $-0,39 * * *$ & $-0,42^{* * *}$ & $-0,40 * * *$ \\
\hline Hohe Bildung & $0,41 * * *$ & $0,44 * * *$ & $0,40 * * *$ \\
\hline \multicolumn{4}{|l|}{ Interaktion mit Geschlecht } \\
\hline Fokusvariable*Frauen & $-0,07^{*}$ & --- & --- \\
\hline \multicolumn{4}{|l|}{ Interaktion mit Alter } \\
\hline Fokusvariable*14 bis 29 Jahre & --- & --- & $-0,15^{* * *}$ \\
\hline Fokusvariable*30 bis 49 Jahre & --- & --- & $-0,10 * *$ \\
\hline Fokusvariable*65 Jahre und älter & --- & --- & --- \\
\hline Pseudo $\mathrm{R}^{2}$ & 0,06 & 0,06 & 0,06 \\
\hline Anzahl gültiger Fälle & 27.862 & 27.885 & 28.009 \\
\hline
\end{tabular}

Quelle: FWS 2014, ungewichtet, eigene Berechnungen (DZA). Kontrolliert für subjektives Einkommen, subjektive Gesundheit, Werthaltung Solidarität sowie Design-Variablen (Bundesland, Kontakt über Festnetz/Mobilfunk). ${ }^{*} p<0,05,{ }^{* *} p<0,01,{ }^{* *} p<0,001,---$ nicht signifikant. 


\subsection{Hat der soziale Zusammenhalt im Wohnviertel einen Einfluss darauf, sich zu engagieren? Oder ist nur die individuelle soziale Einbettung wichtig?}

In diesem Abschnitt gehen wir der Frage nach, ob ein guter sozialer Zusammenhalt in der unmittelbaren Wohnumgebung die Wahrscheinlichkeit erhöht, eine freiwillige Tätigkeit auszuüben, wenn man für Unterschiede hinsichtlich Bildung, subjektiver Gesundheit, finanziellen Ressourcen und solidarischer Werthaltung kontrolliert. Wir vermuten, dass der Effekt des Wohnumfelds besonders für ältere Menschen ausgeprägt ist, weil diese eventuell aufgrund eingeschränkter Mobilität stärker auf nahe gelegene Möglichkeiten zum Engagement angewiesen sein könnten. Da die wahrgenommene Gemeinschaftlichkeit im Wohnumfeld vermutlich mit den Indikatoren der individuellen sozialen Einbettung zusammenhängt, kontrollieren wir in der multivariaten logistischen Regression nicht nur für Geschlecht, Alter und Bildung, sondern auch für die Netzwerkqualität, das Unterstützungspotenzial auBerhalb des Haushaltes und das soziale Vertrauen.

Die Ergebnisse der Regressionsanalyse sind in Tabelle 19-7 dargestellt. Die abhängige Variable ist Engagement (ja/nein). Engagement wird vorhergesagt mit dem Zusammenhalt im Wohnviertel, allen drei Aspekten sozialer Einbettung sowie subjektivem Einkommen, subjektiver Gesundheit, Werthaltung Solidarität sowie Bundesland und Kontakt über Festnetz/Mobilfunk. Zusätzlich wird noch für Geschlecht, Alter und Bildung kontrolliert und es werden die Interaktion von Geschlecht und Alter mit dem Zusammenhalt im Wohnviertel betrachtet. Als Referenzgruppe für die Testung der Interaktionen wurde auch hier die Gruppe der Männer im Alter von 50 bis 64 Jahren gewählt.

Die multivariate Analyse zeigt auch nach Berücksichtigung von gesundheitlichen und finanziellen Ressourcen und Unterschieden in Werthaltungen und Bildung, dass alle Aspekte der sozialen Einbettung - also Netzwerkqualität (Koeffizient 0,20), Unterstützung außerhalb des Haushaltes (Koeffizient 0,52) und das soziale Vertrauen (Koeffizient 0,13) - unabhängig voneinander und signifikant mit der Wahrscheinlichkeit zusammenhängen, sich zu engagieren. Über den Einfluss der individuellen Einbettung hinaus ist der Zusammenhang mit dem sozialen Zusammenhalt im Wohnviertel statistisch bedeutsam (Koeffizient 0,10). Unabhängig davon wie gut ein Mensch individuell sozial eingebunden ist gilt, dass je höher der Zusammenhalt im unmittelbaren Wohnumfeld ist, desto höher die Wahrscheinlichkeit, sich zu engagieren. Der Einfluss des Wohnviertels ist gleich stark für Frauen und Männer und Personen ab 30 Jahren. Nur die jüngste Altersgruppe bildet eine Ausnahme: Für diese Personengruppe hat die Gemeinschaftlichkeit im Wohnviertel keine Auswirkungen auf die Engagementrate (Koeffizient -0,11). 
Tabelle 19-7: Ergebnisse einer logistischen Regression, Vorhersage freiwilligen Engagements durch Zusammenhalt im Wohnviertel, Netzwerkqualität, Unterstützungspotenzial, Soziales Vertrauen, Geschlecht, Alter, Bildung sowie Interaktionen des Zusammenhaltes mit Geschlecht und Alter, 2014

\begin{tabular}{|c|c|}
\hline & Engagement (nein/ja) \\
\hline Zusammenhalt im Wohnviertel & $0,10 * * *$ \\
\hline Netzwerkqualität & $0,20 * * *$ \\
\hline Unterstützungspotenzial & $0,52 * * *$ \\
\hline Soziales Vertrauen & $0,13^{* * *}$ \\
\hline \multicolumn{2}{|l|}{ Geschlecht (Referenz: Männer) } \\
\hline Frauen & $-0,25 * * *$ \\
\hline \multicolumn{2}{|l|}{ Alter (Referenz: 50 bis 64 Jahre) } \\
\hline 14 bis 29 Jahre & --- \\
\hline 30 bis 49 Jahre & --- \\
\hline 65 Jahre und älter & $-0,24 * * *$ \\
\hline \multicolumn{2}{|l|}{ Bildung (Referenz: Mittlere Bildung) } \\
\hline Noch Schüler/in & $0,54 * * *$ \\
\hline Niedrige Bildung & $-0,38 * * *$ \\
\hline Hohe Bildung & $0,40 * * *$ \\
\hline \multicolumn{2}{|l|}{ Interaktion mit Geschlecht } \\
\hline Zusammenhalt*Frauen & --- \\
\hline \multicolumn{2}{|l|}{ Interaktion mit Alter } \\
\hline Zusammenhalt*14 bis 29 Jahre & $-0,11^{* *}$ \\
\hline Zusammenhalt*30 bis 49 Jahre & --- \\
\hline Zusammenhalt*65 Jahre und älter & --- \\
\hline Pseudo $\mathrm{R}^{2}$ & 0,06 \\
\hline Anzahl gültiger Fälle & 27.378 \\
\hline
\end{tabular}

Quelle: FWS 2014, ungewichtet, eigene Berechnungen (DZA). Kontrolliert für subjektives Einkommen, subjektive Gesundheit, Werthaltung Solidarität sowie Design-Variablen (Bundesland, Kontakt über Festnetz/Mobilfunk). ${ }^{*} p<0,05$, $* * p<0,01, * * * p<0,001,---$ nicht signifikant. 


\subsection{Ist die individuelle soziale Einbettung besser je länger man sich engagiert hat?}

Bislang haben wir in diesem Kapitel in der Hauptsache darüber berichtet, welchen Einfluss die individuelle und kontextuelle soziale Einbettung für das Engagement haben kann. Aber natürlich ist es andersherum genauso denkbar, dass sich das soziale Eingebundensein eines Menschen durch seine freiwillige Tätigkeit erhöht. In einer querschnittlichen Analyse lassen sich diese beiden denkbaren Wirkrichtungen nicht voneinander trennen. Doch sollten die Möglichkeiten zum sozialen Austausch, die häufig mit freiwilligen Tätigkeiten verbunden sind, die individuelle soziale Einbettung verbessern, kann man erwarten, dass Menschen, die länger engagiert sind, über eine bessere soziale Integration verfügen als Personen, die weniger lang freiwillig tätig sind. Diesen möglichen Zusammenhang haben wir innerhalb der Gruppe der engagierten Personen untersucht.

Tabelle 19-8 stellt die Ergebnisse von drei logistischen Regressionen dar. Die abhängigen Variablen sind Netzwerkqualität (hoch/niedrig), Unterstützungspotenzial außerhalb des Haushaltes (ja/nein) und soziales Vertrauen (hoch/ niedrig). Der interessierende Prädiktor ist die Engagementdauer, gemessen in Jahren, die als metrische Variable in das Modell eingefügt wurde. Langjährige freiwillige Tätigkeiten von über zehn Jahren Dauer wurden zu einer Kategorie ,mehr als zehn Jahre Engagement ' zusammengefasst. Voranalysen haben ergeben, dass bei einer Dauer von über zehn Jahren keine weiteren $\mathrm{Zu}$ wächse in der sozialen Einbettung zu beobachten waren, die über die vorangegangenen jährlichen Zunahmen hinausgingen. Neben dem reinen Effekt der Dauer interessierten uns die Interaktionen der Engagementdauer mit Geschlecht und Alter. Sollte eine solche vorliegen, würde es den Schluss zulassen, dass verschiedene gesellschaftliche Gruppen hinsichtlich ihrer sozialen Einbettung unterschiedlich von einem Engagement profitieren. Um die Einflüsse von Drittvariablen auszuschließen, berücksichtigten wir auch Unterschiede in der Bildung, subjektivem Einkommen, subjektiver Gesundheit und der Werthaltung Solidarität.

Die drei Modelle zeigen, dass sich die Wahrscheinlichkeiten, über Unterstützungspotenzial außerhalb des Haushaltes zu verfügen oder ein hohes soziales Vertrauen zu haben, mit steigender Engagementdauer nicht erhöhen. Allerdings geht die Dauer des Engagements mit einer höheren Wahrscheinlichkeit einher, über eine hohe Netzwerkqualität zu verfügen (Koeffizient 0,05 ). Dieser Befund unterstützt unsere Vermutung, dass die Möglichkeiten zum sozialen Austausch, die häufig Teil einer freiwilligen Tätigkeit sind, Menschen helfen können, ein qualitativ hochwertiges persönliches Netzwerk aufzubauen. Wir finden keine signifikanten Interaktionen der Dauer mit dem Geschlecht oder der Altersgruppe. Dies bedeutet, dass der Einfluss der Dauer des Engagements auf die individuelle soziale Einbettung für alle beobachteten Gruppen gleich stark ist. 
Tabelle 19-8: Ergebnisse logistischer Regressionen, Vorhersage von Netzwerkqualität, Unterstützungspotenzial, Soziales Vertrauen durch Dauer des Engagements, Geschlecht, Alter, Bildung sowie Interaktionen der Dauer mit Geschlecht und Alter - nur Engagierte, 2014

\begin{tabular}{|c|c|c|c|}
\hline & Netzwerkqualität & Unterstützungspotenzial & Soziales Vertrauen \\
\hline Dauer des Engagements & $0,05 * * *$ & --- & --- \\
\hline \multicolumn{4}{|c|}{ Geschlecht (Referenz: Männer) } \\
\hline Frauen & $0,29 * *$ & $0,34 *$ & --- \\
\hline \multicolumn{4}{|c|}{ Alter (Referenz: 50 bis 64 Jahre) } \\
\hline 14 bis 29 Jahre & --- & $0,67 * *$ & $-0,57^{* * *}$ \\
\hline 30 bis 49 Jahre & --- & --- & $-0,39 * * *$ \\
\hline 65 Jahre und älter & --- & --- & --- \\
\hline \multicolumn{4}{|c|}{ Bildung (Referenz: Mittlere Bildung) } \\
\hline Noch Schüler/in & $0,35^{*}$ & $-0,74 * * *$ & $0,37 * * *$ \\
\hline Niedrige Bildung & $-0,26 * *$ & --- & $-0,27^{* * *}$ \\
\hline Hohe Bildung & $0,48 * * *$ & --- & $0,50 * * *$ \\
\hline \multicolumn{4}{|l|}{ Interaktion mit Geschlecht } \\
\hline Dauer*Frauen & --- & --- & --- \\
\hline \multicolumn{4}{|l|}{ Interaktion mit Alter } \\
\hline Dauer*14 bis 29 Jahre & --- & --- & --- \\
\hline Dauer $* 30$ bis 49 Jahre & --- & --- & --- \\
\hline Dauer*65 Jahre und älter & --- & --- & --- \\
\hline Pseudo $\mathrm{R}^{2}$ & 0,05 & 0,03 & 0,06 \\
\hline Anzahl gültiger Fälle & 12.184 & 12.147 & 12.191 \\
\hline
\end{tabular}

Quelle: FWS 2014, ungewichtet, eigene Berechnungen (DZA). Kontrolliert für subjektives Einkommen, subjektive Gesundheit, Werthaltung Solidarität sowie Design-Variablen (Bundesland, Kontakt über Festnetz/Mobilfunk). ${ }^{*} p<0,05$, $* * p<0,01, * * * p<0,001,---$ nicht signifikant.

\subsection{Fazit}

Freiwilliges Engagement und individuelle soziale Einbettung sind in verschiedener Hinsicht miteinander verwoben. Auf der einen Seite geht ein gutes soziales Netzwerk mit größeren Möglichkeiten einher sich zu engagieren. Soziale Unterstützung kann helfen, eine freiwillige Tätigkeit weiterzuverfolgen, auch wenn man einmal anderweitige Schwierigkeiten bewältigen muss. Und das Vertrauen in andere Menschen kann die
Wahrscheinlichkeit erhöhen, ein Engagement zu beginnen, weil es die Bereitschaft erhöht, einen Beitrag für die Gemeinschaft zu leisten. Auf der anderen Seite kann eine freiwillige Tätigkeit Möglichkeiten bieten mit anderen Menschen in Kontakt zu kommen und langfristige soziale Beziehungen aufzubauen. In der Politikwissenschaft wird zudem häufig argumentiert, dass gerade die Beteiligung an freiwilligen Tätigkeiten 
das soziale Vertrauen fördert (Kaina 2004). In unseren Analysen finden wir Hinweise für beide Wirkrichtungen zwischen sozialer Einbettung und freiwilligem Engagement.

Die Wahrscheinlichkeit sich zu engagieren steigt, wenn Personen über ein gutes Netzwerk verfügen, wenn sie auf Unterstützung außerhalb des eigenen Haushaltes zurückgreifen können und wenn sie anderen Menschen im Allgemeinen vertrauen. Wie unsere multivariaten Analysen zeigen, wirken diese unterschiedlichen $\mathrm{Fa}$ cetten der individuellen Einbettung unabhängig voneinander. Dieser Befund gibt einen Hinweis darauf, dass verschiedene Facetten unterschiedliche Ressourcen bereitstellen, die auf differenzierte Weise das Engagement beeinflussen.

Subjektive Netzwerkqualität: Das individuelle Netzwerk kann Anreize geben, sich überhaupt erst einer freiwilligen Tätigkeit zu widmen. Informationen über das Engagement können beispielsweise von Freundinnen, Freunden oder Bekannten vermittelt werden. Manche Menschen werden auch direkt von Menschen aus ihrem Netzwerk aufgefordert, sich zu engagieren (Tang 2006; siehe Kapitel 15). Gerade unter diesem Blickwinkel ist es interessant, dass unsere Analysen zeigen, dass das Engagement von Frauen weniger stark mit der persönlichen Netzwerkqualität in Zusammenhang steht als das Engagement von Männern. Hinsichtlich ihres Engagements profitieren Frauen also weniger als Männer von sozialen Kontakten. Über die Ursachen dieses Befundes lässt sich an dieser Stelle nur spekulieren. Es sind jedoch zwei Möglichkeiten zur Interpretation denkbar. Zum einen besteht das soziale Netzwerk von Frauen in der Regel zu einem großen Teil ebenfalls aus Frauen. Frauen engagieren sich im Durchschnitt weniger häufig, deshalb mögen Anreize aus einem weiblichen Netzwerk, sich zu engagieren, weniger häufig sein als aus den Netzwerken von Männern. Zum anderen könnte es eine Rolle spielen, dass wenn man Menschen direkt anspricht, ob sie eine freiwillige Tätigkeit ausüben wollen, vermutlich die Überlegung eine Rolle spielt, ob diese Person in der Lage ist eine solche auszuführen. Vielleicht werden Männer häufiger als Frauen als geeignet für ein Engagement wahrgenommen. Gegen diese Interpretation spricht allerdings, dass es keine Altersgruppenunterschiede im Zusammenhang zwischen der Netzwerkqualität und den Engagementraten gibt, obwohl auch ältere Menschen häufig als weniger fähig angesehen werden als jüngere Personen. Trotzdem könnte es sich lohnen, die negativen Geschlechterstereotypen in Bezug auf das Engagement weiter zu erforschen, um Stereotype bewusstzumachen und abzubauen und damit die Zahl der Frauen, die sich engagieren, zu erhöhen. Nichtdestotrotz könnte der Zusammenhang zwischen der Qualität des Netzwerks und dem Engagement auch andersherum begründet sein. Möglicherweise ist es die in der Gruppe ausgeübte freiwillige Tätigkeit, die das persönliche Netzwerk beeinflusst. Wir zeigen, dass mit jedem Jahr, in dem sich ein Mensch engagiert, die Wahrscheinlichkeit zunimmt, mit seiner persönlichen sozialen Einbettung zufrieden zu sein. Dieser Befund spricht dafür, dass ein gutes individuelles Netzwerk mehr als nur eine Voraussetzung für Engagement ist. Das soziale Miteinander, das oft den Rahmen einer freiwilligen Tätigkeit bildet, kann auch die subjektiv erlebte soziale Einbettung fördern, und das sogar unabhängig von Geschlecht und Alter. Forschungsergebnisse zu den schädlichen Effekten von Einsamkeit zeigen, wie wichtig die subjektiv wahrgenommene soziale Einbettung für die Gesundheit und das persönliche Wohlergehen nicht nur im Alter, sondern im gesamten Lebensverlauf ist. Empfinden Personen ihre individuellen sozialen Beziehungen als ungenügend, kann das Gefühl entstehen, einsam zu sein (Hawkley \& Cacioppo 2010). Einsamkeit ist nicht nur eine gefühlsmäßig stark belastende Erfahrung. Sie ist zudem mit Gesundheitsrisiken verbunden, die in ihrer Stärke vergleichbar sind mit den schädlichen Einflüssen von Rauchen, Übergewicht und Bluthochdruck (Luo, Hawkley, Waite \& Cacioppo 2012). Freiwilliges Engagement kann also über seine Wirkung auf das individuelle soziale Netzwerk hinaus generell ein protektiver Faktor in der lebenslangen Entwicklung sein. 
Soziale Unterstützung: Wir untersuchen den $\mathrm{Zu}-$ sammenhang zwischen dem Engagement und dem Vorhandensein von Hilfe außerhalb des eigenen Haushaltes vor dem Hintergrund der Überlegung, soziale Unterstützung könnte helfen, ein Engagement aufrechtzuerhalten. Für das Vorhandensein sozialen Unterstützungspotenzials fanden wir Unterschiede zwischen Menschen, die sich engagieren, und denen, die es nicht tun. Menschen, die auf ein Unterstützungspotenzial zurückgreifen können, engagieren sich häufiger als Personen, die nicht über ein solches verfügen. Doch die Dauer des Engagements hat keinen Einfluss auf die Wahrscheinlichkeit über Unterstützung zu verfügen. Diesen Umstand kann man vorsichtig so deuten, dass die Existenz von Hilfe außerhalb des eigenen Haushaltes eher für die Aufnahme oder auch die Beibehaltung eines Engagements förderlich ist, als dass sie sich aus der Ausübung der freiwilligen Tätigkeit ergibt. Das muss nicht heißen, dass sich aus dem Engagement keine Beziehungen ergeben würden, die auch Hilfe bei persönlichen Problemen leisten. Man muss bei der Interpretation des Ergebnisses bedenken, dass der prozentuale Anteil an Menschen mit sozialer Unterstützung außerhalb des eigenen Haushaltes sehr hoch ist. Einen Engagementeffekt würde man deshalb wohl eher in der Anzahl der Personen finden, die im individuellen Notfall tatsächlich Hilfe leisten, als im reinen Vorhandensein außerhäuslicher Unterstützung. Trotz des allgemein hohen Niveaus scheint sich die Abwesenheit dieser sozialen Unterstützungsquelle bei Frauen stärker negativ auf das Engagementverhalten auszuwirken als bei Männern. Allerdings finden wir diesen Unterschied nicht, wenn wir für Geschlechterunterschiede in persönlichen Ressourcen kontrollierten. Dieser Befund widerspricht aber nicht der Annahme, dass Frauen möglicherweise stärker auf Unterstützung für ihr Engagement angewiesen sind. Im Durchschnitt verfügen insbesondere ältere Frauen über weniger Bildung und individuelle Ressourcen und Frauen sind in einem stärkeren Ausmaß als Männer doppelt belastet durch Familie und Beruf. Vielleicht können Männer deshalb die Abwesenheit von Unterstützung außerhalb des Haushaltes gut ausgleichen und ihr Engagement auch in persönlichen Krisenzeiten fortsetzen.

Soziales Vertrauen: Wir finden, je höher die individuelle Überzeugung ist, man könne anderen Menschen vertrauen, desto größer ist die Wahrscheinlichkeit, sich zu engagieren. In diesem Zusammenhang ist es besonders interessant, dass unsere multivariaten Analysen ergeben, dass Vertrauen auch dann Engagement vorhersagt, wenn man Unterschiede in solidarischen Werthaltungen berücksichtigt (siehe Kapitel 18). Die Unterschiede zwischen Personen bei der Wichtigkeit des Wertes, anderen Menschen zu helfen, erklären also nicht den gefundenen Zusammenhang zwischen Vertrauen und Engagement. In der Politikwissenschaft geht man davon aus, dass ein hohes soziales Vertrauen mit einer größeren Verbundenheit mit gesellschaftlichen Aufgaben einhergeht (Kaina 2004). Ein hohes soziales Vertrauen kann die individuelle Entscheidung, ein Engagement aufzunehmen, beeinflussen, weil es möglicherweise die subjektive Sinnhaftigkeit eines Engagements erhöht. Unter diesem Blickwinkel ist es besonders interessant, dass ein hohes Maß an Vertrauen die Wahrscheinlichkeit einer freiwilligen Tätigkeit stärker bei älteren Menschen als bei jüngeren Menschen erhöht. Alterspsychologische Theorien gehen davon aus, dass es eine Herausforderung des Überganges in den Ruhestand ist, sich neue sinnstiftende Betätigungsfelder zu suchen (Freund, Nikitin \& Ritter 2009; van Solinge \& Henkens 2008). Personen kurz nach dem Ruhestandsübergang mit hohem sozialen Vertrauen könnten freiwillige Tätigkeiten eher als sinnstiftend empfinden als Menschen mit niedrigerem Vertrauen, deshalb wenden sie sich vielleicht eher als andere dem Engagement nach dem Verlassen des Erwerbslebens zu. Natürlich ist auch diese Interpretation spekulativ und man muss bedenken, dass es die freiwilligen Tätigkeiten sein könnten, die das soziale Vertrauen insbesondere bei älteren Personen erhöhen. Unsere Untersuchungen können jedoch keinen direkten Hinweis darauf 
finden, dass die Ausübung eines Engagements das Vertrauen in andere Menschen verstärkt. Die Engagementdauer steht in keinem Zusammenhang mit der Zustimmung zu der Aussage, den meisten Menschen könne man vertrauen. Dieser Befund stimmt mit den Ergebnissen einer Metaanalyse von längsschnittlichen Studien aus verschiedenen Ländern überein (van Ingen \& Bekkers 2013). Die Autoren kamen zu dem Schluss, dass soziales Vertrauen zwar eine Voraussetzung für Engagement sein kann, Auswirkungen freiwilliger Tätigkeiten auf das Vertrauen jedoch in der Regel kaum nachweisbar seien.

Zusammenhalt im Wohnviertel: Über den Effekt der individuellen sozialen Einbettung und den Einflüssen des Geschlechts, des Alters und von Unterschieden in individuellen Ressourcen und solidarischen Werthaltungen hinaus geht ein hoher sozialer Zusammenhalt in der Wohnumgebung mit einer erhöhten Engagementrate einher. Um sich zu engagieren, ist also nicht nur die persönliche Vernetzung wichtig, der regionale soziale Kontext spielt ebenso eine Rolle. Aber es gilt auch das Gegenteil: In einem Wohnviertel mit schlechtem sozialem Zusammenhalt sind freiwillige Tätigkeiten trotz möglicherweise guter individueller Netzwerke im Durchschnitt weniger häufig. Die Qualität der sozialen Beziehungen in der Wohnumgebung kann also sowohl eine Erleichterung als auch ein Hindernis für das persönliche Engagement darstellen. Entgegen unseren Erwartungen ist es nicht nur die älteste Altersgruppe, deren Engagement vom sozialen Wohnumfeld mitbestimmt wird. Der Einfluss der Wohnumgebung auf das Engagement ist in allen Altersgruppen über 30 Jahren gleich hoch. Nur in der jüngsten Gruppe der 14- bis 29-Jährigen hat das unmittelbare soziale Umfeld keinen Einfluss auf die Wahrscheinlichkeit sich zu engagieren. Vermutlich sind die jüngsten Personen im Durchschnitt nicht nur aufgrund ihrer körperlichen Verfassung mobiler, sondern haben zusätzlich weniger familiäre und berufsbedingte Verpflichtungen als andere Altersgruppen. Deshalb können sie vielleicht weitere Wege in Kauf nehmen, um eine freiwillige Tätigkeit wahrzunehmen.

Implikationen: Die individuelle soziale Einbettung steht in einem starken Zusammenhang mit dem Engagement. Je besser die Einbettung, desto größer die Wahrscheinlichkeit, sich zu engagieren. Das bedeutet auch, dass gesellschaftliche Gruppen wie Menschen mit geringer Bildung, bereits aus diesem Grund geringere $\mathrm{Zu}$ gangsmöglichkeiten $\mathrm{zu}$ freiwilligen Tätigkeiten haben. Zusätzlich profitieren Frauen bezüglich des Engagements weniger stark von ihrem persönlichem Netzwerk als Männer. Das Gemeinschaftsgefühl auf nachbarschaftlicher Ebene zu erhöhen könnte in diesem Zusammenhang ein Weg sein, Zugangsmöglichkeiten für Frauen und sozial weniger stark eingebundene Gruppen zu steigern. Denn der soziale Zusammenhalt in der unmittelbaren Wohnumgebung erhöht die Wahrscheinlichkeit eines Engagements unabhängig von Bildung sowie sozialen, finanziellen und gesundheitlichen Ressourcen.

Außerdem finden wir Hinweise darauf, dass freiwillige Tätigkeiten, wenn sie über einen längeren Zeitraum ausgeübt werden, die individuelle soziale Einbettung erhöhen können. Dazugehören, sozial eingebunden sein ist ein fundamentales menschliches Bedürfnis (Baumeister \& Leary 1995). Menschen aller Altersgruppen brauchen regelmäßigen sozialen Austausch, der in einem Kontext wechselseitiger Wertschätzung stattfindet, um sich wohl zu fühlen. Doch nicht nur die emotionale Seite persönlicher Eingebundenheit ist wichtig für die individuelle Lebensqualität. Soziale Beziehungen stellen häufig die Ressourcen zur Verfügung, mit denen Menschen die Widrigkeiten des Lebens meistern (Uchino, Bowen, Carlisle \& Birmingham 2012). Viele Untersuchungen haben gezeigt, dass die Unterstützung, die wir von anderen Menschen erhalten, das ganze Leben lang ein wichtiges Element für die individuelle Gesundheit und ein positives Lebensgefühl ist (Antonucci 2001). Vielleicht ist es daher sinnvoll gerade um solche Personengruppen für freiwillige Tätigkeiten $\mathrm{zu}$ werben, die vom Risiko bedroht sind, sozial ausgeschlos- 
sen $\mathrm{zu}$ sein. Wenn es gelingt, diese Menschen langfristig für eine freiwillige Tätigkeit zu inte- ressieren, könnte dies den negativen Folgen von Einsamkeit vorbeugen.

\section{Literatur}

Ajrouch, K. J., Blandon, A. Y., \& Antonucci, T. C. (2005). Social networks among men and women: the effects of age and socioeconomic status. The Journals of Gerontology, Series B Psychological Sciences \& Sociologcal Scienes, 60B(6), S311-S317.

Antonucci, T. (2001). Psychology of social support. In: N. J. Smelser \& P. B. Baltes (Hrsg.) International encyclopedia of the social \& behavioral sciences; Bd. 1 (S. 14465-14469). Amsterdam: Elsevier.

Antonucci, T. C., Ajrouch, K. J., \& Birditt, K. S. (2014). The convoy model: explaining social relations from a multidisciplinary perspective. The Gerontologist, 54(1), 82-92.

Baumeister, R. F., \& Leary, M. R. (1995). The Need to Belong: Desire for Interpersonal Attachments as a Fundamental Human Motivation. Psychological Bulletin, 117(3), 497-529.

Bronenbrenner, U. (1977). Toward an experimental ecology of human-development. American Psychologist, 32(7), 513-531.

Dury, S., Willems, J., De Witte, N., De Donder, L., Buffel, T., \& Verté, D. (2014). Municipality and Neighborhood Influences on Volunteering in Later Life. Journal of Applied Gerontology, 1-26.

Freund, A. M., Nikitin, J., \& Ritter, J. O. (2009). Psychological Consequences of Longevity. Human Development, 52(1), $1-37$.

Goth, U. S., \& Småland, E. (2014). The role of civic engagement for men's health and well being in Norway - a contribution to public health. International Journal of Environmental Research and Public Health, 11(6), 6375-6387.

Hawkley, L. C., \& Cacioppo, J. T. (2010). Loneliness Matters: A Theoretical and Empirical Review of Consequences and Mechanisms. Annals of Behavioral Medicine, 40(2), 218-227.

Huxhold, O., Fiori, K. L., \& Windsor, T. D. (2013). The Dynamic Interplay of Social Network Characteristics, Subjective Well-Being, and Health: The Costs and Benefits of Socio-Emotional Selectivity. Psychology and Aging, $28(1), 3$.

Ingen, E. van, \& Bekkers, R. (2013). Generalized Trust Through Civic Engagement? Evidence from Five National Panel Studies. Political Psychology, 36(3), 277-294.

Kaina, V. (2004). Vertrauen in Eliten und die politische Unterstützung der Demokratie. Politische Vierteljahresschrift, 45(4), 519-540.

Litwin, H. (2011). The association between social network relationships and depressive symptoms among older Americans: what matters most? International Psychogeriatrics, 23(6), 930-940.

Luo, Y., Hawkley, L. C., Waite, L. J., \& Cacioppo, J. T. (2012). Loneliness, Health, and Mortality in Old Age: A National Longitudinal Study. Social Science \& Medicine, 74(6), 907-914.

Morrow-Howell, N., Hong, S.-I., \& Tang, F. (2009). Who benefits from volunteering? The Gerontologist, 49, 91-102.

Scheibe, S., \& Carstensen, L. L. (2010). Emotional aging: recent findings and future trends. The Journals of Gerontology, Series B: Psychological Sciences \& Sociologcal Scienes, 65B(2), S135-S144.

Solinge, H. van, \& Henkens, K. (2008). Adjustment to and satisfaction with retirement: Two of a kind? Psychology and Aging, 23(2), 422-434.

Son, J., \& Wilson, J. (2012). Volunteer Work and Hedonic, Eudemonic, and Social Well-Being. Sociological Forum, 27(3), 658-681.

Svendsen, G. L. H., Svendsen, G. T., \& Graeff, P. (2012). Explaining the emergence of social trust: Denmark and Germany. Historical Social Research, 37(3), 351-367.

Tang, F. (2006). What resources are needed for volunteerism? A life course perspective. Journal of Applied Gerontology, $25,375-390$. 
Uchino, B. N., Bowen, K., Carlisle, M., \& Birmingham, W. (2012). Psychological pathways linking social support to health outcomes: A visit with the "ghosts" of research past, present, and future. Social Science \& Medicine, 74(7), 949-957.

Wilson, J., \& Musick, M. (1997). Who Cares? Toward an Integrated Theory of Volunteer Work. American Sociological Review, 62(5), 694-713.

Wrzus, C., Hänel, M., Wagner, J., \& Neyer, F. (2013). Social network changes and life events across the life span: a meta-analysis. Psychological Bulletin, 139(1), 53-80.

Open Access Dieses Kapitel wird unter der Creative Commons Namensnennung 4.0 International Lizenz (http://creativecommons.org/licenses/by/4.0/deed.de) veröffentlicht, welche die Nutzung, Vervielfältigung, Bearbeitung, Verbreitung und Wiedergabe in jeglichem Medium und Format erlaubt, sofern Sie den/die ursprünglichen Autor(en) und die Quelle ordnungsgemäß nennen, einen Link zur Creative Commons Lizenz beifügen und angeben, ob Änderungen vorgenommen wurden.

Die in diesem Kapitel enthaltenen Bilder und sonstiges Drittmaterial unterliegen ebenfalls der genannten Creative Commons Lizenz, sofern sich aus der Abbildungslegende nichts anderes ergibt. Sofern das betreffende Material nicht unter der genannten Creative Commons Lizenz steht und die betreffende Handlung nicht nach gesetzlichen Vorschriften erlaubt ist, ist für die oben aufgeführten Weiterverwendungen des Materials die Einwilligung des jeweiligen Rechteinhabers einzuholen. 